\title{
Gene-based therapies for the induction of spinal fusion
}

\author{
Gregory A. Helm, M.D., Ph.D., Hayan Dayoub, M.D., and John A. Jane, Jr., M.D. \\ Departments of Neurosurgery and Biomedical Engineering, University of Virginia, Charlottesville, \\ Virginia
}

\begin{abstract}
Numerous mesenchymal growth factors with osteogenic properties have now been identified. Although many of these proteins can induce bone formation when delivered on a carrier matrix, these approaches have not been fully developed in the laboratory or clinic. The expression of osteogenic proteins via direct or ex vivo gene therapy techniques is also compelling because high-level, long-term gene expression can now be achieved using novel viral and nonviral vectors. In this brief review the authors will highlight recent advances in genetic therapies for the induction of osteogenesis, as well as their potential use for the promotion of spinal arthrodesis.
\end{abstract}

KeY WORDS - gene therapy $・$ spinal fusion

The potential use of DNA transfer techniques is a theoretically attractive approach for the induction of spinal arthrodesis. It has been shown in numerous studies that viral vectors can be used to implant osteoinductive growth factor genes directly into the paraspinal musculature or into cells that are subsequently implanted adjacent to the spine to enhance the activation and differentiation of pluripotent stem cells to produce mature bone. ${ }^{1,16,23,29}$ The use of gene therapy to upregulate bone growth factor production is theoretically attractive because long-term, tissue-specific, inducible rhGF production may have distinct advantages over direct protein delivery. Although BMPs are probably the most potent proteins for inducing osteogenesis, other polypeptides may also be clinically relevant, including TGF- $\beta$, acidic and basic fibroblast growth factors, insulinlike growth factors 1 and 2, and PDGF.

\section{GENE-BASED THERAPIES}

\section{Bone Morphogenetic Proteins}

The BMP field began in the 1960s when Urist ${ }^{34}$ demonstrated that demineralized bone matrix could promote endochondral bone formation when implanted ectopically in rodents. His research laboratory subsequently demonstrated that this osteogenic activity was induced by lowmolecular weight glycoproteins isolated from bone and that these proteins could promote bone formation when delivered to ectopic or orthotopic locations. ${ }^{35}$ With the advancement of molecular biology techniques, Wozney, et al. ${ }^{37}$ and Celeste, et al., ${ }^{8}$ cloned the first BMPs. Bone morphogenetic proteins have now been demonstrated to com-

\footnotetext{
Abbreviations used in this paper: $\mathrm{BMP}=$ bone morphogenetic proteins; $\mathrm{CT}=$ computerized tomography $\mathrm{PDGF}=$ platelet-derived growth factor; rhBMP = recombinant human BMP; rhGF = recombinant human GF; TGF- $\beta=$ transforming growth factor $-\beta$.
}

prise a large number of proteins within the TGF- $\beta$ superfamily, which have similarities in their amino acid sequences. ${ }^{8,37}$ These proteins display a remarkable range of physiological activity and play a major role in the growth and development of numerous organ systems, including the heart, kidney, gonads, liver, skeleton, ligaments, tendons, and skin. ${ }^{30}$ These proteins bind to specific transmembrane receptors, which function as serine/threonine kinases, to phosphorylate members of the Smad second messenger family. ${ }^{12,13}$ These activated second messengers subsequently translocate to the nucleus, where they regulate the transcription of a variety of genes involved in diverse physiological functions such as cellular migration, proliferation, and differentiation. Bone morphogenetic proteins have not only been shown to be essential for normal skeletal development, but the proteins and their receptors have also been found in osseous regeneration in fracture repair. In numerous studies authors have now demonstrated that BMPs, delivered on biological and nonbiological matrices, can successfully treat long bone fractures and induce spinal fusions in rodents, rabbits, dogs, and primates. ${ }^{5,10,18}$ Boden, et al., ${ }^{5}$ have also demonstrated that rhBMP-2 can successfully induce spinal fusions in humans. In a preliminary study, 14 patients received interbody fusion cages filled with autogenous bone graft or with rhBMP-2 on a collagen carrier. A shorter hospital stay was demonstrated in the rhBMP-2-treated case group compared with the autograft group, and in all patients in the rhBMP group solid fusions were radiographically demonstrated at 6 months. It is becoming clear that rhBMPs will have a major impact on the treatment of spinal instability in the future.

\section{Transforming Growth Factor- $\beta$}

Transforming growth factor $-\beta$ also has a stimulatory effect on bone regeneration, and it has been extensively 


\section{G. A. Helm, H. Dayoub, and J. A. Jane, Jr.}

studied in the field of bone repair. ${ }^{7}$ Similar to the BMPs, TGF- $\beta$ stimulates mesenchymal stem cell proliferation and differentiation. It also promotes angiogenesis, inhibits procollagenase, promotes extracellular matrix deposition, and is chemotactic for fibroblasts and macrophages. Its overall osteoinductive potential is fairly weak compared with some of the osteoinductive BMPs. Transforming growth factor- $\beta$ like the BMPs, forms heterotetromeric complexes with their appropriate serine/threonine kinase receptors, which subsequently transphosphorylate Smad intracellular second messengers. ${ }^{12,13}$ Numerous studies have been performed to examine the effects of TGF- $\beta$ in bone fracture models. Although the effects of TGF- $\beta$ are difficult to compare among these studies because of differences in experimental animal models, delivery techniques, and differences in the delivered TGF- $\beta$ isoforms, it appears that TGF- $\beta$ does have the ability to promote healing of fractures but certainly not as impressively as BMPs. ${ }^{24,27}$

\section{Platelet-Derived Growth Factor}

The production of PDGF begins with the expression of two polypeptide chains called A and B. ${ }^{33}$ The expression of these chains in cells leads to the production of three molecules, PDGF-AA homodimers, PDGF-BB homodimers, and PDGF-AB heterodimers. It appears as though PDGF-AA acts as a local osteogenic growth factor, whereas PDGF-BB and PDGF-AB act in a systemic fashion. Platelet-derived growth factor is typically synthesized by a variety of cells, including monocytes, macrophages, and platelets. It has a strong stimulatory effect on mesenchymal cells, including chondroblasts and osteoblasts. For example, it stimulates osteoblast proliferation, as well as collagenous and noncollagenous protein synthesis. ${ }^{20}$ Several research groups have been studying PDGF as it relates to the healing of normal fractures and also as an osteogenic growth factor to stimulate osteoinduction. Andrew, et al., ${ }^{3}$ performed biopsy sampling of 16 fracture sites at various times after injury, and they evaluated the specimens by PDGF immunohistochemistry and in situ hybridization. Platelet-derived growth factor-A chains were found to be expressed in endothelial cells and mesenchymal cells at the injury site, whereas the PDGF-B chain was found to be localized in osteoblasts. The findings of this study demonstrate that PDGF may play an important regulatory role in fracture repair. In a separate study, Nash, et al. ${ }^{26}$ demonstrated in rabbits that PDGF delivered on a collagen carrier had clear osteogenic activity compared with collagen alone. In the PDGF-treated animals significantly more callous formation was demonstrated compared with control animals; this led to improved biomechanical strength in the PDGF-treated group. The osteogenic potential of PDGF is currently being harnessed clinically through the use of synthetic graft materials treated with platelet-rich plasma, which has high levels of PDGF, TGF- $\alpha$, and TGF- $\beta{ }^{25}$ This interesting protein clearly displays unique activity, which may be combined with other growth factors to improve osteoinduction in the clinical setting. Gene therapy techniques used to induce the expression of the aforementioned genes in regions requiring bone regeneration may prove to be an excellent approach to promote bone formation in a variety of clinical situations.

\section{BACKGROUND OF GENE THERAPY}

Somatic gene therapy is a strategy in which specific genes are inserted into target cells to alter their genetic repertoire for therapeutic purposes. Although initial clinical trials on gene-based therapy have demonstrated limited success, the potential use of genetic therapeutics continues to excite basic scientists and clinical researchers in virtually every field of medicine. ${ }^{6,15}$ The common difficulties that typically occur in gene therapy studies include inadequate cellular transduction rates and insufficient transgene expression. Currently, both viral and nonviral vectors are being developed as potential gene delivery vehicles. In this review, we will discuss only the major viral vectors, which include adenoviruses, adeno-associated viruses, retroviruses, and herpes viruses.

\section{Adenoviral Vectors}

Adenoviruses are double-stranded DNA viruses that bind to specific cell surface receptors and enter cells by endocytosis, and the contents of the virus are then released into the cytoplasm. ${ }^{21,32}$ Genes within the virus are divided into immediate-early genes, early genes, and late genes according to the period in which the genes are expressed. The immediate-early genes activate early gene transcription, the early genes are involved both in subsequent viral replication and host immune evasion, and the late genes code for structural proteins of the virus. ${ }^{21}$ In most adenoviral vectors studies to date the vectors are derived from the adenovirus serotype 5, which is rendered replication defective by deletion of certain early genes, typically the E1 region.

The advantages of adenoviral vectors include: the ability to produce high titers of virus; its low toxicity; its extrachromosomal life cycle, which reduces the risk of insertional mutagenesis; and its ability to transduce a wide variety of cells. ${ }^{21,22}$ The virus can also accommodate up to $8 \mathrm{~kb}$ of foreign DNA. Its disadvantages stem in part from its advantages. Because the virus does not integrate into the cellular genome, the duration of gene expression is limited, and therapeutic genes are not passed to the progeny of the transduced cells. In addition, humoral and cellular immune responses at the adenoviral vector treatment site can markedly diminish transgene expression levels. Research is currently being undertaken to attenuate further the host immune responses against the adenoviral vectors, such as deleting the viral DNA polymerase gene ( $\Delta$ pol adenoviral vectors) or completely eliminating the viral genome in the adenoviral vector (gutless adenoviral vectors).

\section{Adeno-Associated Viral Vectors}

The use of adeno-associated viral vectors for the application of BMP gene therapy is a compelling approach. ${ }^{14}$ For several reasons the adeno-associated virus vector, which is a defective single-stranded parvovirus, is a unique and attractive vector for gene therapy studies: it integrates stably into cellular genomes; has the ability to transduce a variety of host cells; maintains high levels of gene expression; and can transfect both proliferating and quiescent cells. Its ability to integrate without causing any 
long-term toxicity, to be relatively heat and $\mathrm{pH}$ stable, and to be generated at high titers also makes it ideally suited for gene transfer. Numerous studies have demonstrated that adeno-associated vectors can efficiently transduce muscle and other cells in vivo, causing little inflammatory response. Fisher, et al., ${ }^{14}$ have reported that adeno-associated virus lacZ can clearly transduce muscle cells in mice and that its genome is efficiently incorporated into the nuclear region, where it is maintained as head-to-tail concatamers. Adeno-associated virus vectors seem to insert at single integration sites, and interestingly, no cytotoxic T-cell response occurs after vector treatment. Other investigators have subsequently demonstrated that adenoassociated viral vectors can transduce muscle cells with reporter genes, the mouse erthyropoetin gene, the alpha-1 antitrypsin gene, and the cystic fibrosis transmembrane conductance regulator gene in a variety of animals, including rodents, canines, and primates. In an important recent report, Bohl et al., ${ }^{6}$ also demonstrated that the DNA inserted into adeno-associated virus vectors can be large enough to include an inducible promoter, which can be used to regulate transgene expression. Although the production of adeno-associated virus vectors was initially fraught with technical difficulties, current techniques of vector production led to a high yield of recombinant adeno-associated virus that is completely free of wild-type adeno-associated virus.

\section{Retroviral Vectors}

Retroviruses are RNA viruses that enter cells through interactions with viral envelope proteins and cell surface glycoproteins. Once they enter the cytoplasm, retroviral reverse transcriptase produces a double-stranded DNA copy, which subsequently integrates into the host genome during mitosis. The transduced progeny cells all carry the transgene after the integration.

One disadvantage of retroviral vectors is that retroviruses can only integrate their genetic material into proliferating cells. ${ }^{11}$ Because normal tissues adjacent to the spine are relatively quiescent, adequate transduction by direct injection may be difficult to achieve. Another major disadvantage of retroviral vectors are their low rate of infectivity and instability of the virions. To circumvent this, retroviral vectors are typically introduced via vectorproducing cells. These cells, often-modified fibroblasts, can transiently produce larger quantities of virus and improve the transfection rate. In animal tumor models this rate can be increased from less than $5 \%$ to over $50 \%$, although it is thought that the transduction rate in studies of human brain tumor remains low. ${ }^{28}$ The use of retroviruses for the delivery of osteogenic genes will most likely be limited to ex vivo approaches such as for the genetic modification of osteoblastic progenitor cell populations.

\section{Herpes Viral Vectors}

Herpes viruses are double-stranded DNA viruses that can induce significant lesions such as cold sores and encephalitis in humans. Typically, the viruses remain dormant and cause no significant disease. In most previous gene therapy studies the investigators have used herpes viral vectors composed of herpes simplex type 1 . In the normal life cycle of these viruses, the virion fuses with the cell membrane and is transported to the nucleus, where, after several phases of gene transcription, the cell lyses and releases progeny viral particles. Herpes viruses also have the ability to enter a latency phase, during which the viral genome is not actively transcribed. This latency phase can be lost during cell division. ${ }^{21}$

Herpes viral vectors have the advantage of being able to accommodate up to $40 \mathrm{~kb}$ of foreign DNA, and they can infect both proliferating and quiescent cells. Herpes viral vectors can be used to insert foreign DNA into a variety of cell populations, including myocytes with limited toxicity and may, therefore, potentially be a reasonable vector for gene therapy. ${ }^{9}$

\section{GENE THERAPY WITH OSTEOGENIC GF GENES}

Osteogenic growth factor gene therapy has multiple advantages over the direct administration of the therapeutic peptide. Currently, growth factors need to be delivered on biological or nonbiological carrier systems, which may make them difficult to deliver in a minimally invasive fashion. Direct-approach gene therapy techniques have the advantage of being able to express growth factors in target cells continuously, for short or long periods of time, which may improve the osteogenic potential for each specific protein. ${ }^{17}$ In addition, because direct gene therapy approaches can be performed in a percutaneous fashion, they may make delivery less invasive than direct protein/carrier delivery. Genetic therapies also have the capability of expressing BMPs in a regulated fashion by using inducible promoters. Therefore, gene expression can be upregulated or downregulated externally as the clinical situation changes. Further, gene therapy has the capability of expressing proteins in specific cells by using tissue-specific promoters or modified vectors with tissue-specific tropism. Because the costs associated with producing recombinant human proteins remains high, genetic approaches may be not only more effective but also more cost efficient.

Numerous research groups are currently studying BMP gene therapy for the induction of numerous tissues, including bone, cartilage, ligament, and tendon. To date, first generation adenoviral vectors containing the $B M P-2$, $-4,-6$, and -9 genes have successfully induced osteogenesis in rodents. ${ }^{2,36}$ These first-generation vectors, however, consistently induce an immune response, which can severely limit their osteogenic potential. The use of athymic nude rodents or immunosuppression has a positive effect on the volume of bone formation when these vectors are used, indicating that transgene expression may be adequate if the induced immune response can be attenuated. Further advances in vector technology need to be made before adenoviral vectors can be used in the clinical setting. In the paraspinal region, both BMP-2 and BMP-9 gene therapy have successfully induced spinal arthrodesis in athymic nude animals. ${ }^{1,16}$ In these studies, the vector was injected percutaneously into the paraspinal musculature. Within 3 months, a large fusion mass was observed at the injection site, which solidly fused at least two spinal levels. The induced bone was in direct contact with the 


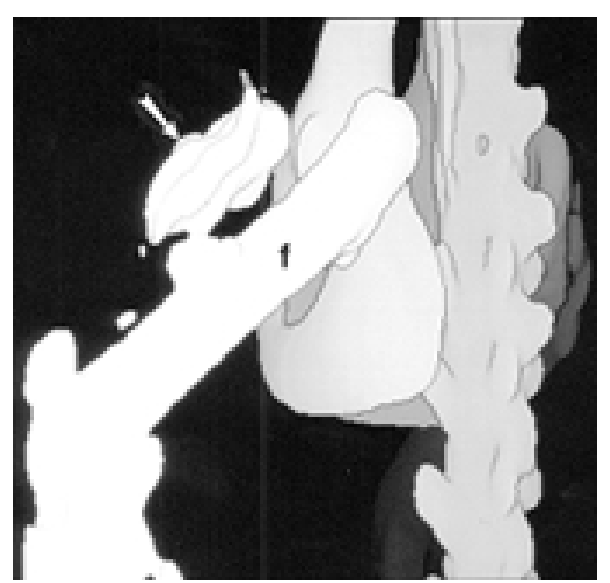

Fig. 1. Three-dimensional reconstructed CT scan of a rodent thigh musculature injected with a gutless adenoviral vector containing the human BMP-9 gene (Ad-gtls-BMP-9), demonstrating ectopic bone formation (arrow) at the treatment site adjacent to the femur (f).

host bone and clearly produced a solid spinal fusion, without evidence of neural compromise in the central canal or lateral regions. Studies are currently underway on gutless adenoviral vectors and adeno-associated viral vectors, which should have a markedly reduced immune response compared with first-generation adenoviral vectors. The three-dimensional reconstructed CT scan in Fig. 1 demonstrates an athymic nude rat thigh injected with a gutless BMP-9 adenoviral vector (Ad-gtls-BMP-9). Evidence of bone formation is clearly evident at the injection site, as early as 3 weeks after treatment. In addition, the threedimensional reconstructed CT scan in Fig. 2 reveals a rodent thigh injected with a BMP-6 adenoviral vector with a partially deleted DNA polymerase gene (Ad-Apol BMP๑), again demonstrating clear evidence of ectopic bone formation. With further development of these less antigenic adenoviral vectors, the novel BMP vectors may be quite useful in the clinical setting.

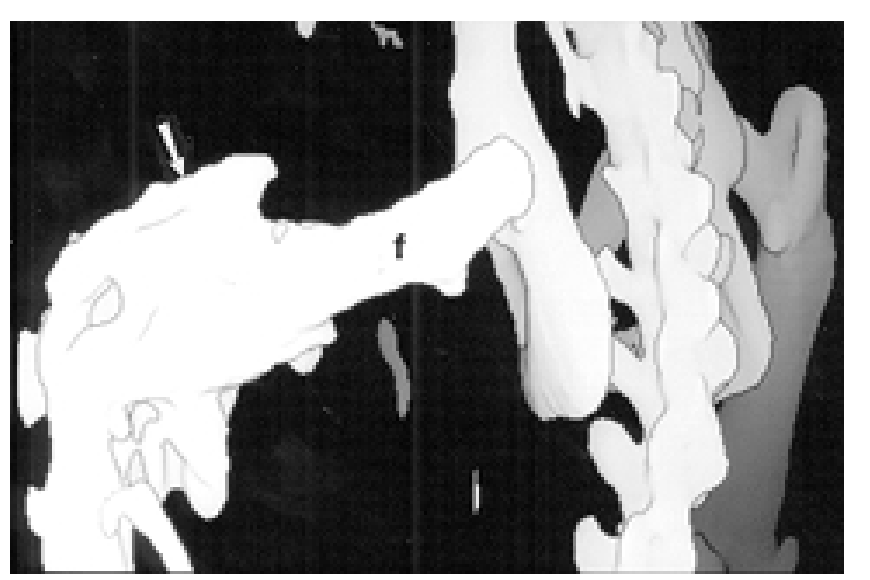

Fig. 2. Three-dimensional reconstructed CT scan of a rodent thigh treated with a BMP-6 adenoviral vector containing a deleted $D N A$ polymerase gene ( $A d-\Delta$ pol-BMP- 6 ), revealing ectopic bone formation (arrow) at the injection site adjacent to the femur.
G. A. Helm, H. Dayoub, and J. A. Jane, Jr.

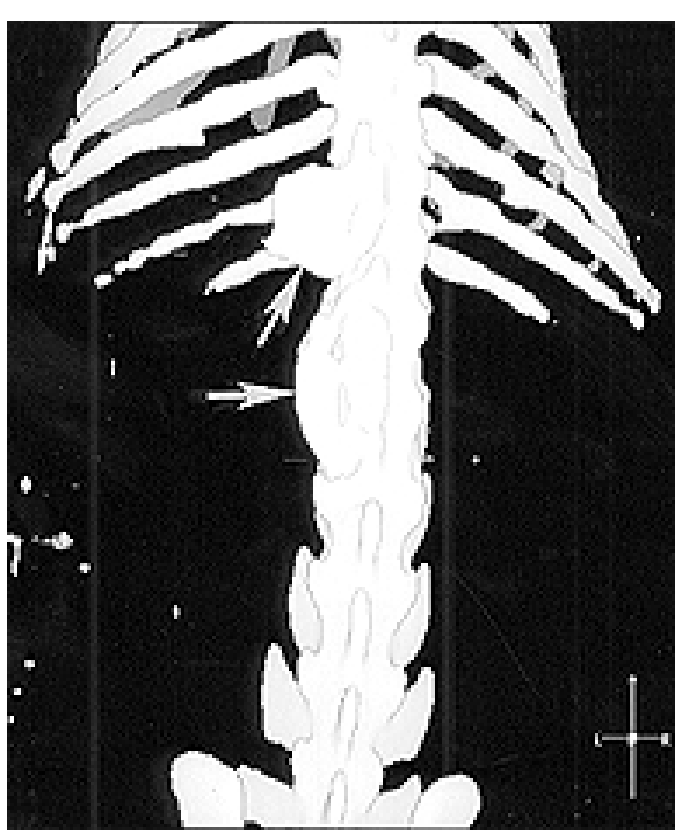

Fig. 3. Three-dimensional reconstructed CT scan of a rodent spine treated with two injections of human mesenchymal stem cells transduced with the human BMP-9 gene via an adenoviral vector. Note the significant bone formation adjacent to the lamina and spinous processes at the treatment sites (arrows).

The authors of several studies have demonstrated that the number of mesenchymal stem cells present in the body markedly decreases with age, most likely accounting for the diminished rates of healing of skeletal fractures seen in elderly patients. In newborns, the number of mesenchymal stem cells in bone marrow is one per 10,000 marrow cells, whereas in the elderly the number drops to one per 2,000,000 marrow cells. ${ }^{31}$ Because many patients requiring bone repair and regeneration are elderly, treatment paradigms aimed at modifying the extracellular environment with growth factors and osteoconductive scaffolds without stem cell replacement may lead to inadequate bone formation. The delivery of genetically modified osteoprogenitor cells on a matrix delivery vehicle at the treatment site will most likely maximize bone deposition in these patients. The isolation, purification, and expansion of osteoprogenitor cell populations have been accomplished in many species including rodent, canine, and human. In several provocative studies, one by Riew, et al., ${ }^{29}$ and one by Lieberman, et al. ${ }^{23}$ the authors have demonstrated that bone marrow-derived mesenchymal stem cells transduced with the $B M P-2$ gene can induce paraspinal bone formation in rodents and rabbits It was subsequently shown in our laboratory that human mesenchymal stem cells transduced with the human $B M P$ 9 transgene via an adenoviral vector can also induce spinal arthrodesis in athymic nude rats (Fig. 3). Although the BMP genes have been the most heavily studied bone morphogen for ex vivo gene therapy, Boden, et al., ${ }^{4}$ have inserted the osteogenic $L M P-1$ gene into allogenic bone marrow cells, also inducing successful spinal fusion in rodents and rabbits. Interestingly, massive amounts of bone formation occurred using relatively low transfection 
rates with the $L M P-1$ gene. It is thought that $L M P-1$ gene expression up-regulates the expression of a variety of other osteogenic growth factors, which, in turn, stimulate bone formation.

In the future, a variety of other approaches could be used to induce strong ectopic or orthotopic bone formation via gene therapy approaches. It appears that BMP heterodimers such as BMP-4/7 and BMP-2/7 may have increased osteogenic potential compared with their homodimeric counterparts. ${ }^{19,30}$ Therefore, the injection of two vectors in combination may strongly improve osteogenic activity. It is anticipated in the near future that the further developments in the field of stem cell therapies, in combination with osteogenic growth factor gene therapy and osteoconductive scaffolding materials, will strongly enhance the rate and quality of bone deposition in the paraspinal region.

\section{References}

1. Alden TD, Pittman DD, Beres EJ, et al: Percutaneous spinal fusion using bone morphogenetic protein-2 gene therapy. J Neurosurg 90:109-114, 1999

2. Alden TD, Pittman DD, Hankins GR, et al: In vivo endochondral bone formation using a bone morphogenetic protein 2 adenoviral vector. Hum Gene Ther 10:2245-2253, 1999

3. Andrew JG, Hoyland JA, Freemont AJ, et al: Platelet-derived growth factor expression in normally healing human fractures. Bone 16:455-460, 1995

4. Boden SD, Titus L, Hair G, et al: Lumbar spine fusion by local gene therapy with a cDNA encoding a novel osteoinductive protein (LMP-1). Spine 23:2486-2492, 1998

5. Boden SD, Zdeblick TA, Sandhu HS, et al: The use of rhBMP2 in interbody fusion cages. Definitive evidence of osteoinduction in humans: a preliminary report. Spine 25:376-381, 2000

6. Bohl D, Salvetti A, Moullier P, et al: Control of erythropoietin delivery by doxycycline in mice after intramuscular injection of adeno-associated vector. Blood 92:1512-1517, 1998

7. Carrington JL, Roberts AB, Flanders KC, et al: Accumulation, localization, and compartmentation of transforming growth factor beta during endochondral bone development. J Cell Biol 107:1969-1975, 1988

8. Celeste AJ, Iannazzi JA, Taylor RC, et al: Identification of transforming growth factor beta family members present in bone-inductive protein purified from bovine bone. Proc Natl Acad Sci USA 87:9843-9847, 1990

9. Coffin RS, Howard MK, Cumming DV, et al: Gene delivery to the heart in vivo and to cardiac myocytes and vascular smooth muscle cells in vitro using herpes virus vectors. Gene Ther 3:560-566, 1996

10. Cook SD, Dalton JE, Tan EH, et al: In vivo evaluation of recombinant human osteogenic protein (rhOP-1) implants as a bone graft substitute for spinal fusions. Spine 19:1655-1663, 1994

11. Culver KW: Gene therapy for malignant neoplasms of the CNS. Bone Marrow Transplant 18 (Suppl 3):S6-S9, 1996

12. Derynck R: TGF-beta-receptor-mediated signaling. Trends Biochem Sci 19:548-553, 1994

13. Derynck R, Zhang Y: Intracellular signalling: the mad way to do it. Curr Biol 6:1226-1229, 1996

14. Fisher KJ, Jooss K, Alston J, et al: Recombinant adeno-associated virus for muscle directed gene therapy. Nat Med 3: 306-312, 1997

15. Graham FL, Prevec L: Methods for construction of adenovirus vectors. Mol Biotechnol 3:207-220, 1995

16. Helm GA, Alden TD, Beres EJ, et al: Use of bone morphogenetic protein-9 gene therapy to induce spinal arthrodesis in the rodent. J Neurosurg 92 (Spine 2): 191-196, 2000
17. Helm GA, Alden TD, Sheehan JP, et al: Bone morphogenetic proteins and bone morphogenetic protein gene therapy in neurological surgery: a review. Neurosurgery 46:1213-1222, 2000

18. Helm GA, Sheehan JM, Sheehan JP, et al: Utilization of type I collagen gel, demineralized bone matrix, and bone morphogenetic protein-2 to enhance autologous bone lumbar spinal fusion. J Neurosurg 86:93-100, 1997

19. Israel DI, Nove J, Kerns KM, et al: Heterodimeric bone morphogenetic proteins show enhanced activity in vitro and in vivo. Growth Factors 13:291-300, 1996

20. Kasperk $\mathrm{CH}$, Wergedal JE, Mohan S, et al: Interactions of growth factors present in bone matrix with bone cells: effects on DNA synthesis and alkaline phosphatase. Growth Factors 3:147-158, 1990

21. Kramm CM, Sena-Esteves M, Barnett FH, et al: Gene therapy for brain tumors. Brain Pathol 5:345-381, 1995

22. Le Gal La Salle G, Robert JJ, Berrard S, et al: An adenovirus vector for gene transfer into neurons and glia in the brain. Science 259:988-990, 1993

23. Lieberman JR, Le LQ, Wu L, et al: Regional gene therapy with a BMP-2-producing murine stromal cell line induces heterotopic and orthotopic bone formation in rodents. J Orthop Res 16:330-339, 1998

24. Lind M, Schumacker B, Soballe K, et al: Transforming growth factor-beta enhances fracture healing in rabbit tibiae. Acta Orthop Scand 64:553-556, 1993

25. Marx RE, Carlson ER, Eichstaedt RM, et al: Platelet-rich plasma: growth factor enhancement for bone grafts. Oral Surg Oral Med Oral Pathol Oral Radiol Endod 85:638-646, 1998

26. Nash TJ, Howlett CR, Martin C, et al: Effect of platelet-derived growth factor on tibial osteotomies in rabbits. Bone 15: 203-208, 1994

27. Nielsen HM, Andreassen TT, Ledet T, et al: Local injection of TGF-beta increases the strength of tibial fractures in the rat. Acta Orthop Scand 65:37-41, 1994

28. Ram Z, Culver KW, Walbridge S, et al: In situ retroviral-mediated gene transfer for the treatment of brain tumors in rats. Cancer Res 53:83-88, 1993

29. Riew KD, Wright NM, Cheng S, et al: Induction of bone formation using a recombinant adenoviral vector carrying the human BMP-2 gene in a rabbit spinal fusion model. Calcif Tissue Int 63:357-360, 1998

30. Sakou T: Bone morphogenetic proteins: from basic studies to clinical approaches. Bone 22:591-603, 1998

31. Service RF: Tissue engineers build new bone. Science 289: 1498-1500, 2000

32. Seth $\mathrm{P}$, Willingham MC, Pastan I: Adenovirus-dependent release of $51 \mathrm{Cr}$ from $\mathrm{KB}$ cells at an acidic $\mathrm{pH}$. J Biol Chem 259: 14350-14353, 1984

33. Solheim E: Growth factors in bone. Int Orthop 22:410-416, 1998

34. Urist MR: Bone: formation by autoinduction. Science 150: 893-899, 1965

35. Urist MR, Nogami H, Mikulski A: A bone morphogenetic polypeptide. Calcif Tissue Res 21 (Suppl):81-87, 1976

36. Varady P, Li JZ, Cunningham M, et al: Morphologic analysis of BMP-9 gene therapy-induced osteogenesis. Hum Gene Ther: 697-710, 2001

37. Wozney JM, Rosen V, Celeste AJ, et al: Novel regulators of bone formation: molecular clones and activities. Science 242: 1528-1534, 1988

Manuscript received March 13, 2001.

Accepted in final form March 30, 2001.

Address reprint requests to: Gregory A. Helm, M.D., Ph.D., Department of Neurosurgery, Box 800212, University of Virginia Health Sciences Center, Charlottesville, Virginia 22908. email: gah9r@virginia.edu. 